\title{
On the use of the Fourier transform to determine the projected rotational velocity of line-profile variable B stars ${ }^{\star}$
}

\author{
C. Aerts ${ }^{1,2}$, S. Simón-Díaz ${ }^{3,4}$, P. J. Groot ${ }^{2}$, and P. Degroote ${ }^{1, \star \star}$ \\ 1 Instituut voor Sterrenkunde, KU Leuven, Celestijnenlaan 200D, 3001 Leuven, Belgium \\ e-mail: Conny.Aerts@ster.kuleuven.be \\ 2 Department of Astrophysics/IMAPP, Radboud University Nijmegen, 6500 GL Nijmegen, The Netherlands \\ 3 Instituto de Astrofísica de Canarias, 38200 La Laguna, Tenerife, Spain \\ ${ }^{4}$ Departamento de Astrofísica, Universidad de La Laguna, 38205 La Laguna, Tenerife, Spain
}

Received 16 April 2014 / Accepted 24 July 2014

\begin{abstract}
Context. The Fourier transform method is a popular tool for deriving the rotational velocities of stars from their spectral line profiles. However, its domain of validity does not include line-profile variables with time-dependent profiles.

Aims. We investigate the performance of the method for such cases, by interpreting the line-profile variations of spotted B stars and of pulsating B stars, as if their spectral lines were caused by uniform surface rotation along with macroturbulence.

Methods. We perform time-series analysis and harmonic least-squares fitting of various line diagnostics and of the outcome of several implementations of the Fourier transform method.

Results. We find that the projected rotational velocities derived from the Fourier transform vary appreciably during the pulsation cycle whenever the pulsational and rotational velocity fields have similar magnitudes. The macroturbulent velocities derived while ignoring the pulsations can vary by tens of $\mathrm{km} \mathrm{s}^{-1}$ during the pulsation cycle. The temporal behaviour of the deduced rotational and macroturbulent velocities are in antiphase with each other. The rotational velocity is in phase with the second moment of the line profiles.

Conclusions. The application of the Fourier method to stars with considerable pulsational line broadening may lead to an appreciable spread in the values of the rotation velocity, and, by implication, of the deduced value of the macroturbulence. These two quantities should therefore not be derived from single snapshot spectra if the aim is to use them as a solid diagnostic evaluating stellar evolution models of slow-to-moderate rotators.
\end{abstract}

Key words. line: profiles - techniques: spectroscopic - stars: massive - stars: rotation - stars: oscillations - stars: evolution

\section{Introduction}

Along with the effective temperature, gravity, and chemical composition of the stellar surface, the rotation velocity of stars has been considered as an important observational diagnostic for evaluating stellar evolution models, particularly in the case of massive stars (Maeder 2009, for an extensive monograph on the theory of stellar evolution including rotation). Unfortunately, the quantity that is usually measured from high-resolution high signal-to-noise spectroscopy is the projected rotation velocity $v \sin i$ instead of the equatorial rotation velocity. Even in the case where the rotation frequency is available with high precision from time series analysis of spectro-polarimetry or from asteroseismology (e.g. Aerts et al. 2014, for a compilation of massive stars with these quantities available), the stellar radius is not within reach of direct observations for large samples of stars. Hence, the outcome of stellar evolution computations is often compared with measured values of $v \sin i$ (e.g. Hunter et al. 2009; Brott et al. 2011; Markova et al. 2014, and references therein). An accurate determination of $v \sin i$ is thus essential to

\footnotetext{
* The spectroscopic time series used in this paper, along with many others, are available from http://newton.ster.kuleuven. be/ roy/helas/

$\star \star$ Postdoctoral Fellow of the Fund for Scientific Research of Flanders (FWO), Belgium
}

test compliance with stellar models. Here, we focus on determining $v \sin i$ for slowly to moderately rotating B-type line-profile variable stars.

Since the development of high-resolution spectroscopy initiated more than three decades ago, it is well known that rotation is not the only line broadening mechanism at work in massive stars. We refer to Smith \& Karp (1978) and Campos \& Smith (1980) for early pioneering work in this topic, where macroturbulent velocities were introduced to obtain good fits to the observed line profiles, while Aerts \& De Cat (2003) and Howarth (2004) presented early reviews in the case of pulsational line broadening of main-sequence B stars and macroturbulent line broadening of O-type stars and BA-type supergiants, respectively. The physical origin of the often high macroturbulent velocities adopted to fit spectral lines of hot stars is still uncertain and may be quite different for various types of objects. This contrasts with the situation for cool stars, where the macroturbulent velocities needed to obtain good spectral lines fits are identified as being due to the limitations of using $1 \mathrm{D}$ model atmospheres rather than considering the convective motions properly in a $3 \mathrm{D}$ description (Asplund et al. 2000). Owing to the unknown physical nature of the macroturbulent velocities in massive stars, the separation of the rotational line broadening from the combination of other broadening mechanisms is far from trivial, particularly if only a single spectrum or a few snapshot spectra are available. In the 
case of pulsating B stars, the rotational velocity can be disentangled from the pulsational and microturbulent broadening from time-resolved spectroscopy that covers the entire beating cycle of the pulsations (e.g., Aerts et al. 2010, Chapter 6). Such data sets are rather scarce and are not available in the case of large sample studies.

With the aim of performing an extensive study of stellar rotation in OB-type stars covering all evolutionary stages, Simón-Díaz \& Herrero (2014, hereafter termed SDH14) reviewed the status of the derivation of $v \sin i$, and along with it of macroturbulence $\left(v_{\text {macro }}\right)$, from high-precision spectroscopy. Their analyses led to the conclusion that adequate values for both $v \sin i$ and $v_{\text {macro }}$ can best be obtained from a line-profile analysis relying on the application of the Fourier transform (FT) method (e.g. Carroll 1933; Smith \& Gray 1976; Dravins et al. 1990; Gray 2005, and references therein), followed by a goodness-offit (GOF) approach to determine $v_{\text {macro }}$ using a radial-tangential approximation for the macroturbulence. Only in the case where $v \sin i$ from the FT and from the GOF approaches agree can one trust the derived values of these two quantities.

As pointed out in SDH14, and also stressed by Sundqvist et al. (2013), the derivation of $v \sin i$ from the first zero of the FT of a spectral line may be quite erroneous for low rotation velocities and in the presence of large macroturbulence, the quoted limit of applicability being $v \sin i=50 \mathrm{~km} \mathrm{~s}^{-1}$ in the case where the line profile is affected by an important macroturbulent contribution. This is particularly the case when the spectral resolution of the instrument is limited. The risk of misinterpreting lineprofile broadening in terms of macroturbulence while ignoring pulsational velocity fields due to gravity modes and their accompanying line broadening has already been stressed by Aerts et al. (2009) from extensive simulations of line-profile variations.

With the current work, we shed new light on the matter by focusing on B stars in the core-hydrogen burning phase and in the regime $v \sin i<80 \mathrm{~km} \mathrm{~s}^{-1}$, keeping in mind that a large fraction of these stars are line-profile variables (e.g. Telting et al. 2006), a fact that tends to be ignored when deriving $v_{\text {macro }}$. We illustrate how various versions of the FT method give rise to a wide range of $v \sin i$ and the deduced $v_{\text {macro }}$ values during the variability cycle for stars whose surface displays inhomogeneities and/or stellar pulsations. It turns out that the estimation of $v \sin i$ from snapshot spectra can be subject to considerable uncertainty when dealing with line-profile variable stars.

\section{Selected line-profile variable B stars}

In this work, we consider a few prototypical well-studied lineprofile variable core-hydrogen burning B stars as the simplest case studies to assess the robustness of the FT method when dealing with asymmetric line profiles. In a future study, a sample of O-type stars and B-type supergiants whose line-profile properties are yet unknown will be considered (Simón-Díaz et al., in prep.).

Line-profile variability of B stars can have several causes. The two most prominent ones are chemical and/or temperature inhomogeneities at the stellar surface giving rise to monoperiodic rotational modulation in the line profiles, or stellar pulsations leading to mono- or multiperiodic profile changes depending on the character of the excited modes. A mixture of these two, i.e. pulsations and surface spots, may also occur (e.g. Telting et al. 1997; Uytterhoeven et al. 2005; Briquet et al. 2012).

Depending on their dominant restoring force, the pulsations are either pressure (p-) modes, gravity (g-) modes, or mixed modes. The $\mathrm{p}$-modes give rise to dominant radial velocity fields while the g-modes lead to dominant tangential velocities. We point out that the pulsations also give rise to temperature variations across the stellar surface (e.g. De Ridder et al. 2002), but that these are only a minor secondary effect compared to the lineprofile variability induced by the pulsational velocity field, even in the case of temperature-sensitive spectral lines (Schrijvers \& Telting 1999). For a detailed derivation and extensive discussion of how spectral line profiles change during p- or g-mode pulsation cycles, we refer to Aerts et al. (2010, Chapter 6).

As appropriate test cases, we consider a few well-studied and bright slowly rotating B stars, either with coherent pulsations excited by a heat mechanism or with chemical peculiarity (Bp stars), thus excluding Be stars and evolved B stars with mass loss. Chemically peculiar Bp stars and pulsating B stars co-exist with constant $\mathrm{B}$ stars during the main sequence phase (e.g. Briquet et al. 2007a). While the distinction between pulsations and rotational modulation as the causes of the variability of slowly rotating stars is difficult to make from light curves (e.g. Degroote et al. 2011), it is easy to do so from the high-resolution, high signal-to-noise spectroscopic time series that we use here. The properties and the considered data sets of the stars under study are summarised in Table 1 and discussed in Sects. 2.1 to 2.3 .

\subsection{Spotted B-type stars}

HD 131120 and HD 105382 were studied extensively by Briquet et al. (2004), by means of high-precision time-series spectroscopy from which the cause of the variability could be unambiguously determined as rotational modulation. Doppler imaging led to detailed abundance surface maps for $\mathrm{Si}$ and $\mathrm{He}$ and to the stellar parameters listed in Table 4 of Briquet et al. (2004), including the rotation frequency, effective temperature, and gravity listed in Table 1. Both stars show two strong and one weak Si spots at their surface and are moderate rotators with $v \sin i=60$ and $70 \mathrm{~km} \mathrm{~s}^{-1}$ for HD 131120 and HD 105382, respectively. In an attempt to find an explanation for the surface spots, a magnetic field was sought for both stars. For HD 105382, such a magnetic field has indeed been detected, both in low- and in high-resolution spectro-polarimetry (Hubrig et al. 2006; Alecian et al. 2011). We are not aware of any magnetic field detection for HD 131120, despite efforts to find one (Briquet et al. 2007b).

\section{2. $\beta$ Cep stars}

We considered three well-known $\beta$ Cep stars in this study. These are stars that pulsate in low-order $\mathrm{p}$ - and g-modes excited by an opacity bump in the partial ionisation layers of iron-like elements that occurs in their envelope at a temperature near $2 \times$ $10^{5} \mathrm{~K}$ (Aerts et al. 2010). Their pulsational velocity vector's radial component is dominant over the tangential one. The first $\beta$ Cep star considered here is HD 16582, which is multiperiodic but has a clear dominant radial mode with moderate amplitude (Aerts et al. 1992, 2006). The second case is the monoperiodic large-amplitude radial pulsator HD 46328 (Saesen et al. 2006) and the third class member is the low-amplitude multiperiodic non-radial pulsator HD 111123 (Aerts et al. 1998; Cuypers et al. 2002). The dominant pulsation frequency and $v \sin i$ of these stars are listed in Table 1, while their full pulsation characteristics are available in the above-mentioned papers. While HD 16582 and HD 111123 do not possess a detectable magnetic field (Aerts et al. 2014), HD 46328 does (Hubrig et al. 2009). None of the three stars shows evidence of detectable rotational modulation in their line profiles. 
C. Aerts et al.: The Fourier transform applied to line-profile variable B stars

Table 1. Stellar parameters and line-profile properties of seven selected variables.

\begin{tabular}{|c|c|c|c|c|c|c|c|}
\hline $\begin{array}{l}\text { Star } \rightarrow \\
\text { Parameter } \downarrow\end{array}$ & HD 131120 & HD 105382 & HD 46328 & HD 16582 & HD 111123 & HD 74195 & HD 181558 \\
\hline$T_{\text {eff }}(\mathrm{K})$ & 18250 & 17400 & 27500 & 23000 & 27000 & 16000 & 14700 \\
\hline $\log g(\mathrm{cgs})$ & 4.1 & 4.2 & 3.8 & 3.8 & 3.7 & 3.9 & 4.2 \\
\hline $\begin{array}{l}\text { Spectral } \\
\text { type }\end{array}$ & B7IIIp & B6III & B0.7IV & B2IV & B0.5IV & B3IV & B5III \\
\hline $\begin{array}{l}\text { Variability } \\
\text { type }\end{array}$ & Si spots & Si spots & $\begin{array}{l}\text { radial } \\
\text { mode }\end{array}$ & $\begin{array}{l}\text { radial } \\
\text { mode }\end{array}$ & $\begin{array}{l}\text { non-radial } \\
\text { p modes }\end{array}$ & $\begin{array}{l}\text { non-radial } \\
\text { g modes }\end{array}$ & $\begin{array}{l}\text { non-radial } \\
\text { g modes }\end{array}$ \\
\hline $\begin{array}{l}\text { Selected } \\
\text { line }\end{array}$ & Si II $4128 \AA$ & Si II $4128 \AA$ & Si III $4552 \AA$ & Si III $4552 \AA$ & Si III $4552 \AA$ & Si II $4128 \AA$ & Si II $4128 \AA$ \\
\hline $\begin{array}{l}v \sin i^{a} \\
\left(\mathrm{~km} \mathrm{~s}^{-1}\right)\end{array}$ & $60 \pm 5^{b}$ & $70 \pm 5^{b}$ & $15.5 \pm 1.5^{c}$ & $1 \pm 1^{d}$ & $18 \pm 5^{e}$ & $20 \pm 3^{e}$ & $9.5 \pm 2.5^{f}$ \\
\hline $\begin{array}{l}\text { Dominant } \\
\text { frequency }\left(\mathrm{d}^{-1}\right)\end{array}$ & 63745 & 0.77214 & 4.77153 & 6.20587 & 5.95867 & 0.35745 & 0.80783 \\
\hline $\begin{array}{l}\text { EW (mÅ) } \\
\text { Range } \\
\text { St.dev. } \\
\langle v\rangle\left(\mathrm{km} \mathrm{s}^{-1}\right)\end{array}$ & $\begin{array}{c}{[84,107]} \\
6.63\end{array}$ & $\begin{array}{c}{[80,105]} \\
6.47\end{array}$ & $\begin{array}{c}{[199,241]} \\
7.37\end{array}$ & $\begin{array}{c}{[136,156]} \\
4.85\end{array}$ & $\begin{array}{c}{[252,293]} \\
6.27\end{array}$ & $\begin{array}{c}{[74,98]} \\
4.38\end{array}$ & $\begin{array}{c}{[100,110]} \\
2.88\end{array}$ \\
\hline $\begin{array}{l}\text { Range } \\
\text { St. dev. } \\
\left\langle v^{2}\right\rangle\left(\mathrm{km}^{2} \mathrm{~s}^{-2}\right)\end{array}$ & $\begin{array}{c}{[-4.70,5.85]} \\
2.93\end{array}$ & $\begin{array}{c}{[-6.77,9.02]} \\
3.77\end{array}$ & $\begin{array}{c}{[-16.19,18.37]} \\
11.52\end{array}$ & $\begin{array}{c}{[-5.16,7.09]} \\
5.15\end{array}$ & $\begin{array}{c}{[-2.98,2.75]} \\
1.12\end{array}$ & $\begin{array}{c}{[-5.33,4.54]} \\
2.12\end{array}$ & $\begin{array}{c}{[-6.66,7.52]} \\
4.58\end{array}$ \\
\hline $\begin{array}{l}\text { Range } \\
\text { St.dev. }\end{array}$ & $\begin{array}{c}{[754,987]} \\
45.8\end{array}$ & $\begin{array}{c}{[987,1496]} \\
98.2\end{array}$ & $\begin{array}{c}{[118,502]} \\
110.1\end{array}$ & $\begin{array}{c}{[113,194]} \\
21.5\end{array}$ & $\begin{array}{c}{[552,871]} \\
58.7\end{array}$ & $\begin{array}{c}{[319,579]} \\
56.8\end{array}$ & $\begin{array}{c}{[64,220]} \\
34.2\end{array}$ \\
\hline $\begin{array}{l}\left\langle v^{3}\right\rangle\left(\mathrm{km}^{3} \mathrm{~s}^{-3}\right) \\
\text { Range } \\
\text { St. dev. }\end{array}$ & $\begin{array}{l}-9177,13887] \\
5740\end{array}$ & $\begin{array}{c}{[-25765,25270]} \\
11413\end{array}$ & $\begin{array}{c}{[-13635,15814]} \\
7741\end{array}$ & $\begin{array}{c}{[-3275,2948]} \\
1861\end{array}$ & $\begin{array}{c}{[-7191,6562]} \\
2254\end{array}$ & $\begin{array}{c}{[-8674,3636]} \\
2305\end{array}$ & $\begin{array}{c}{[-1468,4688]} \\
1539\end{array}$ \\
\hline $\begin{array}{l}\text { 1st zero FT }\left(\mathrm{km} \mathrm{s}^{-1}\right) \\
\text { Range } \\
\text { St. dev. }\end{array}$ & $\begin{array}{c}{[52.7,60.7]} \\
1.60\end{array}$ & $\begin{array}{c}{[43.4,78.8]} \\
6.16\end{array}$ & $\begin{array}{c}{[9.1,17.8]} \\
2.04\end{array}$ & $\begin{array}{c}{[6.8,15.6]} \\
2.29\end{array}$ & $\begin{array}{c}{[15.1,46.1]} \\
8.72\end{array}$ & $\begin{array}{c}{[6.1,27.5]} \\
5.40\end{array}$ & $\begin{array}{c}{[6.3,26.0]} \\
6.13\end{array}$ \\
\hline $\begin{array}{l}\mathrm{V}_{\text {macro }}\left(\mathrm{km} \mathrm{s}^{-1}\right) \\
\text { Range } \\
\text { St. dev. }\end{array}$ & $\begin{array}{c}{[11.1,30.0]} \\
3.64\end{array}$ & $\begin{array}{c}{[10.8,64.2]} \\
10.50\end{array}$ & $\begin{array}{c}{[14.8,21.9]} \\
1.47\end{array}$ & $\begin{array}{c}{[14.8,22.7]} \\
1.73\end{array}$ & $\begin{array}{c}{[29.4,54.3]} \\
6.67\end{array}$ & $\begin{array}{c}{[0.0,22.2]} \\
4.70\end{array}$ & $\begin{array}{c}{[1.3,25.9]} \\
5.54\end{array}$ \\
\hline
\end{tabular}

Notes. The spectral types were taken from the Simbad database. ${ }^{(a)}$ Value deduced from detailed line-profile fitting relying on spot or pulsation models: ${ }^{(b)}$ Briquet et al. (2004); ${ }^{(c)}$ Saesen et al. (2006); ${ }^{(d)}$ Aerts et al. (1992); ${ }^{(e)}$ Briquet \& Aerts (2003); ${ }^{(f)}$ De Cat et al. (2005).

\subsection{Slowly pulsating $B$ stars}

The stars HD 74195 and HD 181558 are two typical cases of multiperiodic g-mode pulsators that are excited by the same opacity mechanism as the $\beta$ Cep stars and better known as slowly pulsating B stars (SPBs; Aerts et al. 2010). The tangential pulsational velocity component of such stars is much greater than the radial component. Their pulsational properties were extensively studied from multicolour photometry and high-precision spectroscopy by De Cat \& Aerts (2002), from which we retrieved the data used for the current study. The main difference between these two stars is that HD 181558 has one dominant g-mode while HD 74195 has several g-modes of similar amplitude. Also, while HD 181558 has no magnetic field detected (Aerts et al. 2014), a weak field seems to be present in HD 74195 (Bagnulo et al. 2012; Hubrig et al. 2013), although it is not accompanied by a detectable signal of rotational modulation.

\subsection{The measured line-profile variations}

For the three hottest pulsators studied here, we considered the Si III line at $4553 \AA$, a well-known spectral line optimally suited to interpret the pulsational variability of $\beta \mathrm{Cep}$ pulsators (Aerts $\&$ De Cat 2003). For the four cooler B stars, we selected the Si II line at $4128 \AA$ (cf. Table 1). For four of the stars in Table 1, the profiles we have selected to work with have been shown in the literature already in the above-mentioned papers, so we do not repeat them all here and refer the reader to the orginal data sources. For three pulsators whose profiles are not yet available in the literature, we show the observations in Fig. 1, along with the profiles of the spotted star HD 131120 to illustrate the contrast in time series between spotted and pulsating B stars. Three of the panels in this figure give the reader a grasp of how stellar pulsations deform the individual line profiles for typical members of pulsation classes whose amplitudes in broad-band photometry are typically between 1 and $35 \mathrm{mmag}$. It can be seen, particularly for the g-mode pulsators, that pulsation modes can strongly affect the line profile shapes, with occurrences of sharp narrow lines at some occasions during the overall pulsation cycle and broad shallow lines at other phases. As illustated in the upper left panel of Fig. 1, the spots on the surface of B stars tend to deform the line profiles mainly in intensity and much less so in velocity.

It is noteworthy that the CoRoT and Kepler space missions have revealed a huge diversity of OB-star photometric variability with a great variety in periodicity and at low levels between $10 \mu \mathrm{mag}$ and $1 \mathrm{mmag}$. While asteroseismology has revealed shortcomings in stellar structure models of B stars from such data (e.g. Degroote et al. 2010), current time-resolved spectroscopy of these stars, which have visual magnitudes between 6 
HD 131120, spotted line-profile variable star

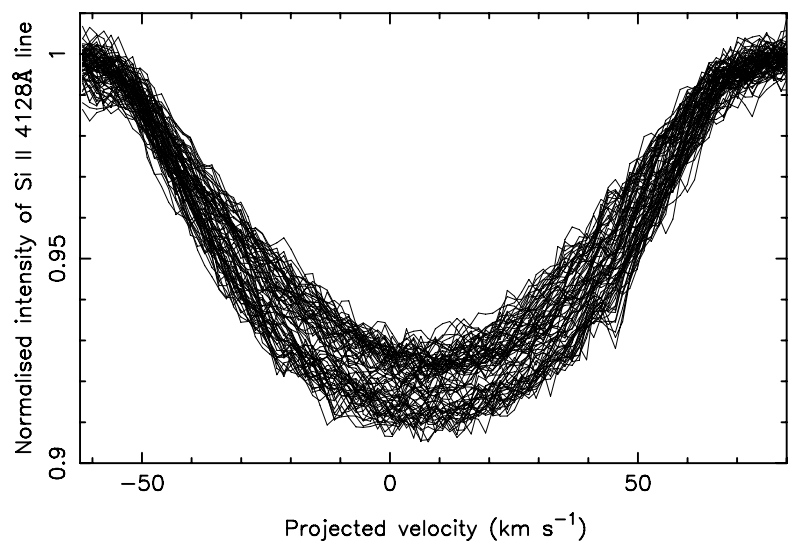

HD 74195, non-radial g-mode pulsator

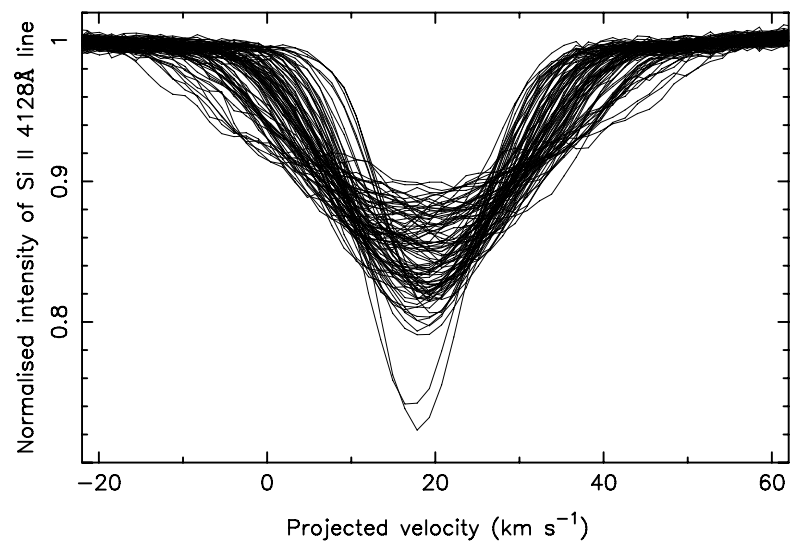

HD 46328, radial pulsator

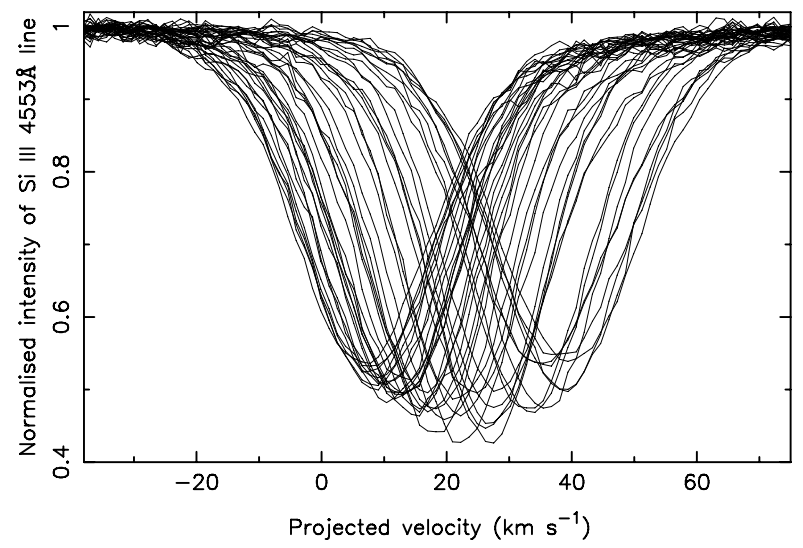

HD 181558, non-radial g-mode pulsator

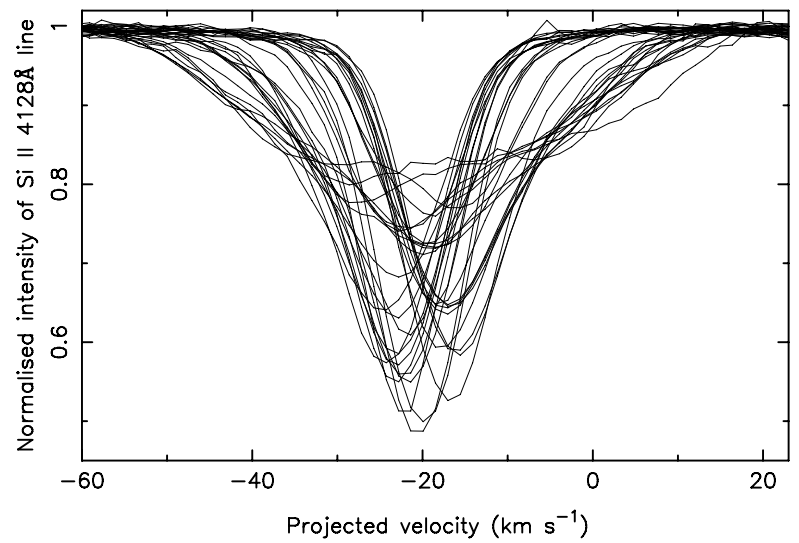

Fig. 1. Line profiles used in this study for four of the targets listed in Table 1. The nature of the variability, the data source, and the laboratory wavelength of the spectral line corresponding to the zero point are discussed in the text. For HD 46328 we only show one tenth of the data for visibility reasons.

and 12, turned out to be of insufficient resolution and signalto-noise to capture the line-profile variability of such lowamplitude pulsations (Degroote et al. 2012).

Appropriate line-profile modelling by means of spots or pulsations requires extensive computations in terms of either spot models or pulsation theory, or both. A prerequisite for this is to identify the character of the spots or the wavenumbers of the pulsation modes, after frequency analysis from line diagnostics. A powerful method of identifying pulsation modes from lineprofile variations in the case of slow- to moderate-rotators is the moment method (Aerts et al. 1992). It is based on the idea, inherited from statistics, that any distribution function is fully characterised if one knows its moments. A theoretical expression was therefore computed for the moments of a line profile based on the eigenvectors and eigenfrequencies deduced from the theory of stellar pulsation. The moments vary periodically in time according to the pulsation modes of the star. It was shown that the three lowest order moments, denoted as $\langle v\rangle,\left\langle v^{2}\right\rangle$, and $\left\langle v^{3}\right\rangle$ and normalised with respect to the equivalent width of the line, are sufficient for identifying the pulsation modes that cause their periodic variability.

The theoretical expressions of the moments for a monoperiodic pulsator are available in Aerts et al. (1992), while those for the generalised version in case of a multiperiodic pulsator are derived in Mathias et al. (1994). The first moment, $\langle v\rangle$, is a measure of the centroid of the line profile and it varies sinusoidally around value zero according to the frequencies of the pulsation modes.
The second moment, $\left\langle v^{2}\right\rangle$, which is a measure of the width of the line profile, varies with the pulsation frequencies and twice their value around a constant value determined by the rotational, pulsational, and intrinsic width of the line. The third moment, which measures the skewness of the profile, varies around value zero according to once, twice, and three times the pulsational frequencies. Mode identification, along with estimation of $v \sin i$ and of the inclination angle $i$, is then achieved by minimising the difference between the measured moment values and those predicted by theory as a function of the wavenumbers $(\ell, m)$ of the modes. For a more detailed explanation of the moment method, we refer to Zima (2008) and Aerts et al. (2010, Chapter 6) ${ }^{1}$. We computed the three moments for all the line profiles of the seven stars. The range of these moment values, as well as the standard deviation of these line diagnostics, are indicated in Table 1. We come back to the temporal variability of the moments in Sect. 4 .

\subsection{Rotational velocities from spot modelling or asteroseismology}

The way to estimate the "true" $v \sin i$ in the case of rotational modulation is to model time-resolved profiles covering the rotation period, assuming spots and/or differential surface rotation.

\footnotetext{
1 The software to perform spectroscopic mode identification and achieve line-profile modelling for non-radial pulsators is freely available from http://www.ster. kuleuven.be/ zima/famias/
} 
For stars cooler than spectral type B, current methods are based on the FT coupled to spot models of differentially rotating stars (e.g. Reiners \& Schmitt 2002, 2003; Ammler-von Eiff \& Reiners 2012, and references therein). For HD 105382 and HD 131120, Briquet et al. (2004) obtained $v \sin i=70 \pm 5$ and $60 \pm 5 \mathrm{~km} \mathrm{~s}^{-1}$, respectively, from modelling of $\mathrm{He}$ and $\mathrm{Si}$ surface spots by assuming constant surface rotation. For the case of pulsators, one must perform spectroscopic mode identification as explained in the previous section. Only when the time series of line-profile variations is modelled properly according to the correct physical processes can one get an appropriate estimate of the true $v \sin i$ of the star, along with the other pulsational velocity parameters.

For the stars in our sample, the $v \sin i$ obtained from spot or pulsation modelling is listed in Table 1. As can be seen, both an under- and an overestimation of $v \sin i$ deduced from the first zero of the FT may occur if one "downgrades" the level of the line-profile modelling by considering only the two parameters $v \sin i$ and $v_{\text {macro }}$ instead of the complete velocity vector due to all the detected and identified pulsation modes.

Given that the cases with time-resolved spectroscopy of sufficient time base and quality to perform the full mode identification are scarce and limited to very bright stars (visual magnitude typically below 6), it is useful to assess the performance of a "downgraded" analysis based on $v \sin i$ and $v_{\text {macro }}$ when the aim is to evaluate stellar evolution models from large samples of stars. For such applications, line-profile variability is usually ignored and one tends to work with snapshot spectra, rather than timeresolved spectroscopy. As shown in Fig. 1 and listed in Table 1, this paper concerns slow- to moderate-rotators; for fast rotators, the pulsational signal in the line profiles gets smeared out by the large rotational broadening whenever the latter begins to dominant the pulsational broadening.

\section{Results of the Fourier transform method}

Following the results of SDH14 and keeping in mind that pulsations lead to radial, as well as tangential velocities, we considered a radial-tangential description of macroturbulence to try and capture the line-profile variability in such a formalism. The time-resolved spectroscopy of the seven stars introduced in the previous section were subjected to the FT method with the aim to determine $v \sin i$, and subsequently $v_{\text {macro }}$, for each of the selected line profiles in the time series. In total we analysed 866 Si line profiles as if spots or pulsations are absent, and we derived $v_{\text {macro }}$ in this approximation.

We first of all used the iacob-broad tool as described in SDH14 to perform the line broadening analysis. This tool has a fixed linear limb-darkening coefficient of 0.6 and makes four sets of computations: $v \sin i$ from the first zero of the FT, $v \sin i$ from a GOF where $v_{\text {macro }}$ is assumed to be zero, $v \sin i$ and $v_{\text {macro }}$ from a GOF assuming both are free parameters, and $v_{\text {macro }}$ resulting from a GOF where the $v \sin i$ is fixed to the value corresponding to the first zero of the FT. In the current version of iacob-broad, the radial and tangential contributions to the macroturbulent velocity are assumed to be equal. In the rest of this paper, we consider the $v \sin i$ from the first zero of the FT derived from iacob-broad as the baseline result.

In addition, we used two other implementations of the FT method by Jankov (1995) and Piters et al. (1996) to assess the robustness of the $v \sin i$ determination from the first zero of the FT of the selected line profile. All three implementations are based on the same principle that the rotational broadening of a spectral line can be distinguished from other additional broadening mechanisms thanks to the property that the convolution of various broadening functions in the wavelength domain transfers into a multiplication in the Fourier domain.

Under the assumptions that one is dealing with uniform surface-rotation, a homogeneous surface temperature and surface chemical composition, and a spherical star with linear limb darkening, $v \sin i$ can in principle be deduced from the FT alone, without detailed modelling of the overall line profile shape. A further assumption is that all occurring broadening mechanisms act independently of each other. Under these circumstances, the zeros of the FT of the profile are inversely proportional to $v \sin i$ (Dravins et al. 1990, for the proportionality coefficients). It is then customary to deduce $v \sin i$ from the first zero of the FT.

The caveat we want to highlight in this work is that the occurrence of surface inhomogeneities or of pulsations introduces line asymmetries, which transform into saddle points or local minima unrelated to $v \sin i$, in the FT of the line profile. This is illustrated for four different profiles observed during the variability cycle for four of the stars in Fig. 2. It can be seen that the "first" zero point not only shifts from one spectrum to another one, but even more importantly that the difference between a saddle point, a local flat minimum, and the first "true" minimum can be hard to judge. This difficulty lies at the origin of inaccurate $v \sin i$ estimates in the case of line-profile variable stars, as we illustrate in the following.

\subsection{Effect of limb darkening on the FT method}

As already stressed by Jankov (1995), the zero points of the FT depend on the limb darkening of the star. Limb-darkening coefficients are available in the literature as a function of $T_{\mathrm{eff}}$, $\log g$, and metallicity of the star, for various wavelength regimes (e.g., Claret \& Bloemen 2011, and references therein). A typical value adopted for early B-type dwarfs in the case of a linear law is $u=0.35$ (Slettebak 1949; Aerts et al. 1992). The temperature varies across the stellar surface and in time due to stellar pulsations. However, as already mentioned above, the changes in line profiles due to pulsations are mainly caused by the local velocity variations on the stellar surface and much less by the local temperature and gravity variations (De Ridder et al. 2002). Moreover, Aerts et al. (1992) investigated the effect of changing the adopted limb darkening when interpreting line-profile variations of hot massive stars and found that the approximation of a constant linear limb-darkening law during the entire pulsation cycle is largely sufficient to interpret the time-series spectroscopy adequately. We thus work in this linear limb darkening approximation in the present paper, but we do show the effect of choosing a different value for the limb darkening coefficient.

In the recent literature, the limb darkening dependence in the derivation of $v \sin i$ has often been ignored in the sense that the limb darkening was either not taken into account (Piters et al. 1996) or was fixed at the "standard" value of $u=0.6$ as adopted by Gray (2005). However, this standard value is only appropriate for cool stars and less so for the hot stars we study here. The effect of limb darkening on the derived value of $v \sin i$ is not negligible but is known. For convenience, we reproduce the table with the first and second zero points of the amplitude of the FT as given by Jankov (1995) in Fig. 3. It can be seen from this figure that the factor between the first zero of the FT for a fully darkened disk and for a constant intensity across the stellar surface amounts to 0.85. This figure, or Table 1 in Jankov (1995), can be used to rescale the stated $v \sin i$-values in the literature that have been derived without limb darkening as in Piters et al. (1996) and Groot et al. (1996) or with a standard value of 0.6 for the linear coefficient as in SDH14, while $u$ should be adapted 
HD 105382, spotted star

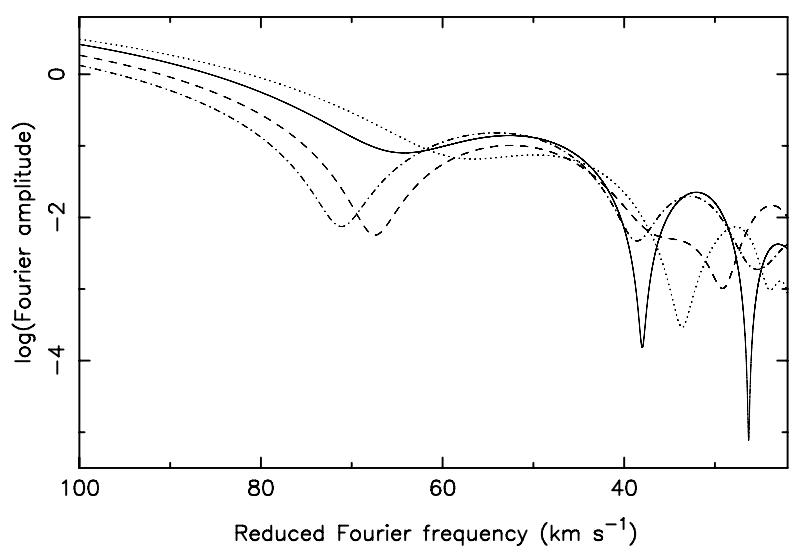

HD 46328, radial pulsator

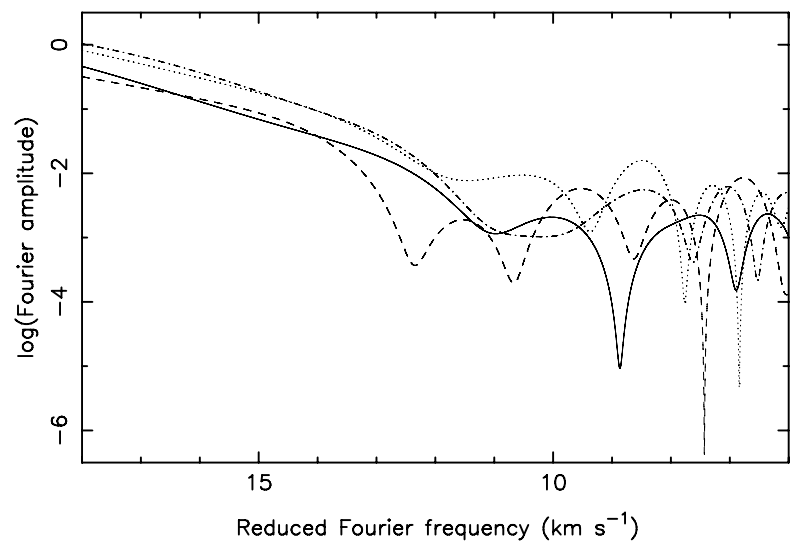

HD 181558, non-radial g-mode pulsator

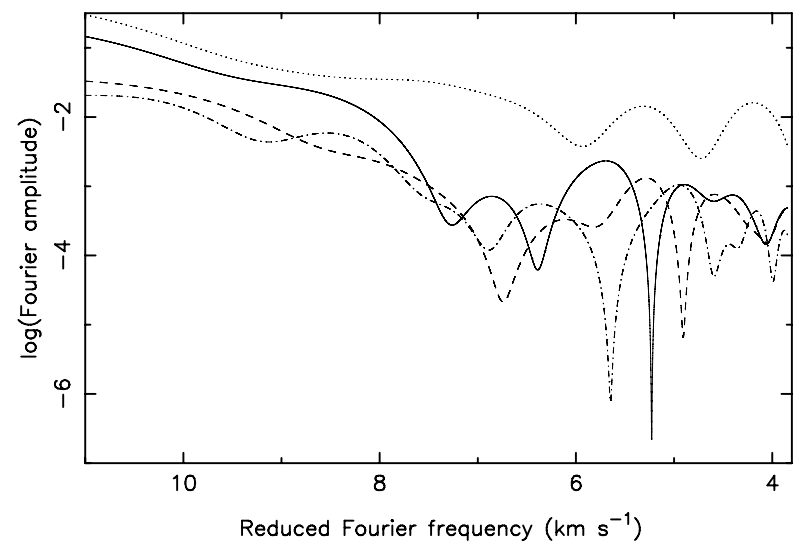

HD 111123, non-radial p-mode pulsator

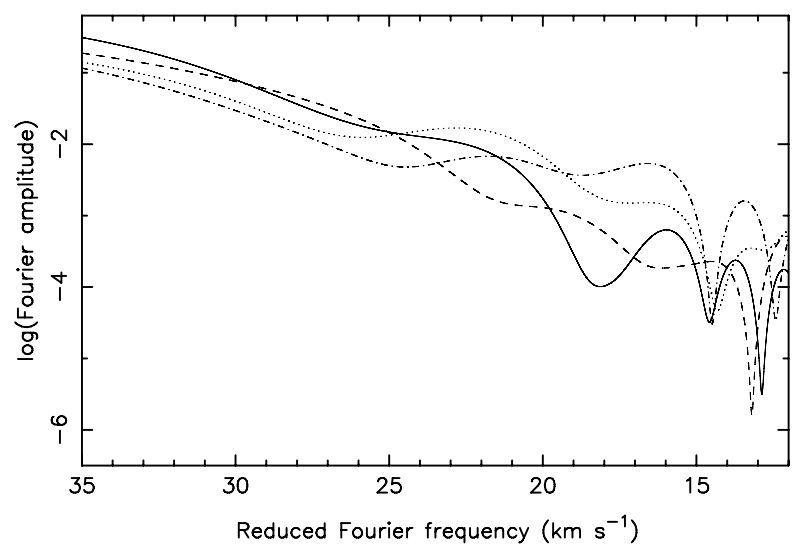

Fig. 2. The Fourier transforms (bottom panels) of four selected profiles (top panels) for four of the stars. The abscissa of the bottom panels shows the FT frequencies transformed to velocity units following Jankov (1995).

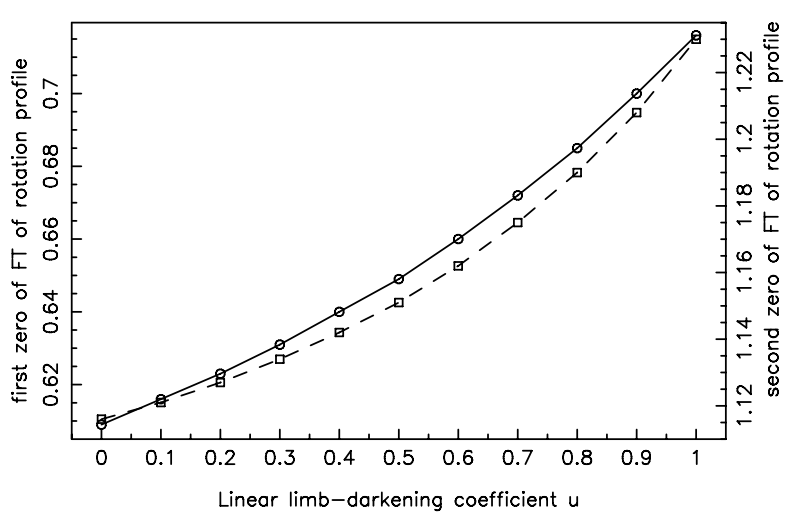

Fig. 3. First (left $y$-axis, full line) and second (right $y$-axis, dashed line) zero points of the amplitude of the FT of a rotation profile as a function of the linear limb-darkening coefficient. Figure produced from Table 1 in Jankov (1995).

to the temperature, gravity and metallicity of the star, as well as to the laboratory wavelength considered. Of these four, the temperature and wavelength are the two most important ones to influence $u$. In early applications (cf. Jankov (1995) for a discussion), the derivation of $v \sin i$ and $u$ from the FT was done simultaneously from the first two zeros. Indeed, the ratio between the first and second zero points as shown in Fig. 3 can be used to derive the most appropriate value of $u$. In modern applications, however, one works the other way around: by fixing the most appropriate value for $u$ according to the temperature of the star and the wavelength of the spectral line, one can assess whether the surface rotation is differential or not (e.g. Reiners \& Schmitt 2003; Ammler-von Eiff \& Reiners 2012, for numerous applications to cool stars).

Given that the linear limb darkening coefficient was fixed to $u=0.6$ in SDH14 and that we want to quantify the effects of stellar pulsations or spots on the derived $v \sin i$ and $v_{\text {macro }}$, we adopted the result from iacob-broad relying on $u=0.6$ for the comparisons between the different implementations of the FT method, but we do show the effect of using a different value of $u$ below as well.

\subsection{The Fourier-Bessel method}

Rather than considering a pure Fourier transform, Piters et al. (1996) used the Fourier-Bessel transform (FBT) of the line profile. The idea behind this is that the Fourier transform of a pure rotational profile, which has an ellipsoidal shape in the absence of limb darkening, is proportional to a first-order Bessel function and that the Bessel transform of this Fourier transform has a maximum at $v \sin i$. The method by Piters et al. (1996) is implemented with the aim of indicating this maximum by the user, from a graphical user interface. The code has the option of being applied to a large number of spectra, as was done and illustrated in Groot et al. (1996) for a sample of F-type stars. 
To compare the results of the FBT with those obtained from the other implementations, we must scale the velocity value derived from the maximum of the FBT, in order to take the effect of limb darkening into account, which we did according to the factor 1.084 as can be deduced from Fig. 3 .

\subsection{Comparison of the results for $v \sin i$}

The authors used three implementations of the $\mathrm{F}(\mathrm{B}) \mathrm{T}$ method to derive $v \sin i$ for all the profiles, independently of each other. This is important to realise, because the results require manual intervention in the sense that the user must select the position of the first extremum. As already said, this is not trivial for the FT method in the cases where saddle points and local minima occur. The iacob-broad and Jankov (1995) tools are different implementations of the same method and were applied by two of the authors independently (SSD and CA) to take the subjectivity of selecting the first minimum into account; the Piters et al. (1996) implementation is less subjective in the choice of the maximum of the FBT because it is less dependent on the line-profile variability, but the exact position of the maximum is less clear and one needs to rescale the result correct for the limb darkening.

We show the comparative results for four of the stars in Fig. 4. We adopted the results of FT using the iacob-broad tool designed by SDH14, where $u=0.6$ was fixed, as the baseline to compare with. In addition to the results from the FT method, we also show the results obtained from a GOF approach to determine $v \sin i$ and $v_{\text {macro }}$ from iacob-broad, as explained in much detail in SDH14. The upper left-hand panel of Fig. 4 shows the results for the spotted star HD 131120 . It can be seen that the FT versus GOF results for $v \sin i$ from iacob-broad (full line versus squares) are in almost perfect agreement except for a few profiles in the time series. There is good agreement with the results from the Jankov (1995) method and also with the results from the FBT method once these are scaled to $u=0.6$ according to Fig. 3. If one keeps in mind that the $v \sin i$ estimate is expected to reach a precision of a few percentage points, then the comparative results for HD 131120 are excellent, keeping in mind that spot modelling led to $v \sin i=60 \pm 5 \mathrm{~km} \mathrm{~s}^{-1}$.

For the large-amplitude radial pulsator HD 46328, the $v \sin i$ values derived from the different codes have quite good agreement, although we find the GOF results to deviate considerably more from the $\mathrm{F}(\mathrm{B}) \mathrm{T}$ results in comparison with the case of a spotted star. This is understandable from the fact that a radial pulsation mode dominantly acts on the central part of the line profile where it introduces broadening and slight skewness due to the radial pulsation velocity, while leaving the outer line wings almost unaltered. The effect of such a profile deformation remains limited in the Fourier transform, but the GOF tries to enforce a good fit to the pulsationally broadened profiles. Deviations from the $v \sin i=15.5 \pm 1.5 \mathrm{~km} \mathrm{~s}^{-1}$ that was obtained from pulsational modelling of the line profiles are quite large for all the FT methods.

The situation is worse for the non-radial multiperiodic p-mode pulsator HD 111123. This is because the non-radial modes lead to broad asymmetric line wings so that saddle points occur in the $\mathrm{F}(\mathrm{B}) \mathrm{T}$, which may imply ambiguity or even an incorrect choice of the position of the first zero point of the FT and uncertainty in the position of the maximum of the FBT. In addition to that, the results from the FT and GOF from iacob-broad differ appreciably, as can be deduced from the spread of the open squares with respect to the full line in the middle panel of Fig. 4. Some estimates are more than a factor two different from the $v \sin i=18 \pm 5 \mathrm{~km} \mathrm{~s}^{-1}$ deduced from appropriate pulsational modelling.

Finally, the results for HD 181558 show yet another pattern, with a clump for the $\mathrm{F}(\mathrm{B}) \mathrm{T}$ methods around a value of $v \sin i \sim$ $8 \mathrm{~km} \mathrm{~s}^{-1}$, except for a few outliers at much higher $v \sin i$ between 15 and $25 \mathrm{~km} \mathrm{~s}^{-1}$ compared to the $v \sin i=9.5 \pm 2.5 \mathrm{~km} \mathrm{~s}^{-1}$ deduced from pulsational modelling. These points correspond to the few profiles in Fig. 1, which are clearly broader than the other ones due to particular beating of the non-radial modes.

Although the FBT method was not designed to provide modelling of the other broadening mechanisms occurring in addition to rotation, particularly not when anisotropic macroturbulence is present (Piters et al. 1996), it does not perform worse in the estimation of the correct $v \sin i$ than the FT method, which is supposed to have good capacities in such situations (Gray 2005).

\section{Temporal behaviour of $v \sin i$ and $v_{\text {macro }}$}

As can be deduced from Fig. 4, the range of $v \sin i$ across the rotation phase of HD 131120 is only $8 \mathrm{~km} \mathrm{~s}^{-1}$, while it reaches about $35 \mathrm{~km} \mathrm{~s}^{-1}$ during the pulsation cycle of the dominant mode for HD 111123. We computed the temporal range and standard deviation of $v \sin i(t)$ and of $v_{\text {macro }}(t)$ for the seven stars and included them in Table 1. A general remark is that we did not treat the microturbulence separately in this work. In this sense, what we list as range of $v_{\text {macro }}(t)$ in Table 1 , is an upper limit for the total amount of turbulent line broadening.

First of all, we come to the conclusion that the $v \sin i(t)$ range over the rotation or pulsation cycle can either be very narrow (HD 131120) or broad (HD 111123) depending on the nature of the spots or pulsations. We cannot provide a general recipe of how $v \sin i(t)$ behaves during the variability cycle from this small sample of stars. Clearly, the range in $v \sin i(t)$ not only depends on the type of variability but is quite different within one class (cf. HD 131120 versus HD 105382).

The range in $v \sin i(t)$ values obtained during the rotation or pulsation cycle has repercussions on the values derived for $v_{\text {macro }}(t)$ as well. We followed the approach of SDH14 to derive the $v_{\text {macro }}(t)$ from either a GOF approach after fixing the $v \sin i(t)$ deduced from the FT method or from a GOF for the two quantities simultaneously. The results are illustrated for four of the seven stars in Fig. 5. It can be seen that the two methods agree very well for the spotted star HD 105382, which had a broad range of $v \sin i(t)$ values resulting from the FT method. For the three pulsators, we find a lower $v \sin i(t)$ value and an accompanying higher $v_{\text {macro }}(t)$ value for the GOF method compared to the FT method. This is logical if one keeps in mind that pulsational broadening during the cycle affects the blue and red line wings in an asymmetrical way (cf. Fig. 1 and Aerts et al. 2010, Chapter 6) and the GOF tries to find the best compromise for the red and blue wings, while spots typically give rise to more local variability in the line, which implies less asymmetry in the line wings (cf. Fig. 1). It has already been stressed in SDH14 and in Sundqvist et al. (2013), as well as in earlier work, that the GOF approach to fitting $v \sin i$ and $v_{\text {macro }}$ simultaneously may lead to a large degeneracy in the parameter space, particularly for slow rotators. This also becomes obvious in our study of the seven stars from Table 2, where we list the maximum uncertainty for $v \sin i(t)$ and for $v_{\text {macro }}(t)$ when determined from a GOF analysis, as well as the uncertainty for these two quantities averaged over the total time series.

We note here in passing that the implementation of Piters et al. (1996) allows the user to "symmetrise" the line profile, by mirroring either the left or the right part with respect to the 


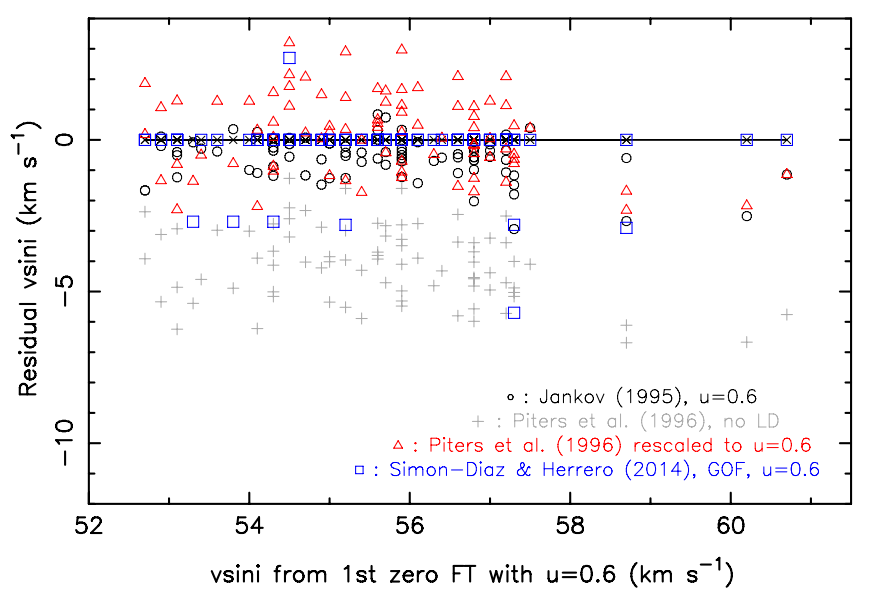

HD 111123, non-radial $p-$ mode pulsator

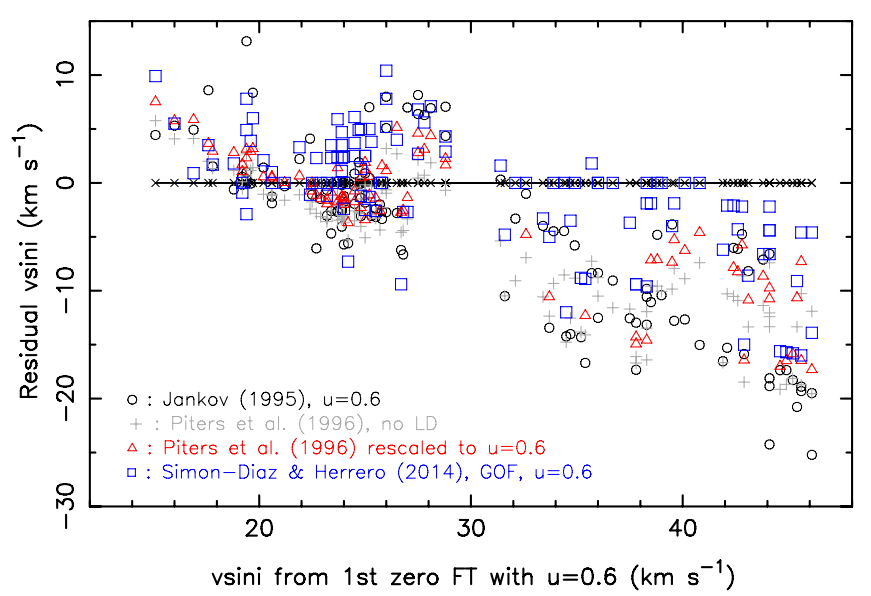

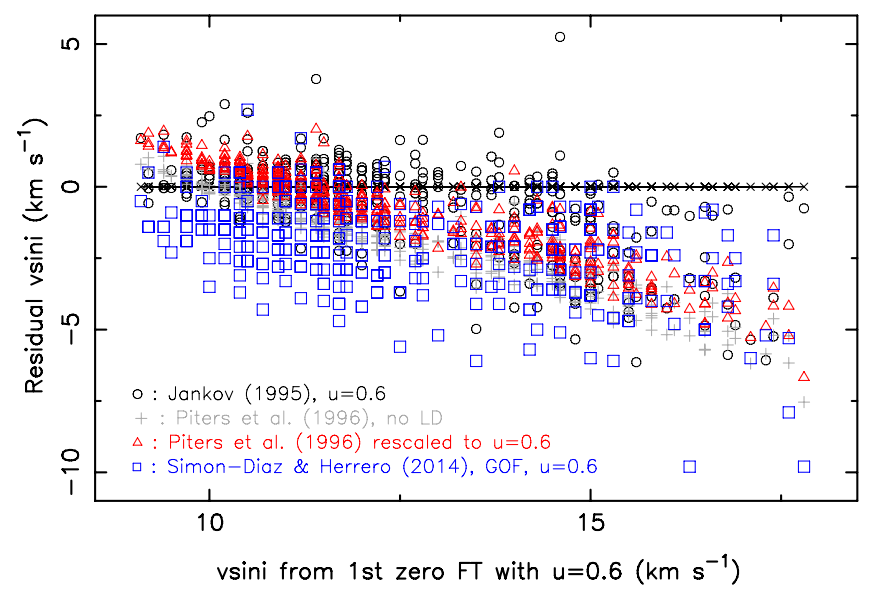

HD 181558, non-radial g-mode pulsator

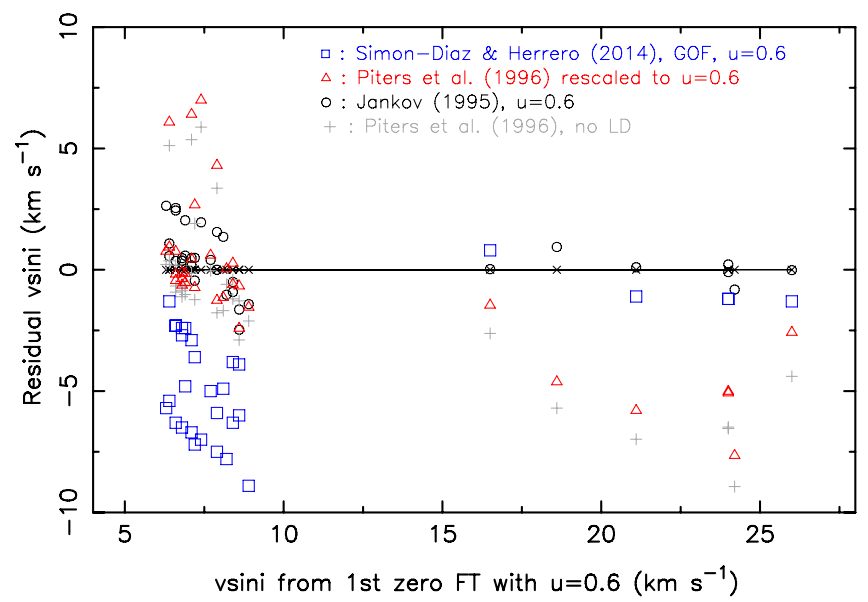

Fig. 4. Comparison between the $v \sin i$ derived from the FT method with $u=0.6$ (full line) and from the FBT and GOF methods as indicated. Colour figure available in the online version of the paper.

Table 2. Maximum error and error averaged over the time series spectroscopy encountered for the $v \sin i$ and $v_{\text {macro }}$ estimates when using the GOF approach to determine both quantities.

\begin{tabular}{|c|c|c|c|c|}
\hline \multirow[t]{2}{*}{ Star } & \multicolumn{2}{|c|}{ Error $v \sin i\left(\mathrm{~km} \mathrm{~s}^{-1}\right)$} & \multicolumn{2}{|c|}{ Error $v_{\text {macro }}\left(\mathrm{km} \mathrm{s}^{-1}\right)$} \\
\hline & Maximum & Average & Maximum & Average \\
\hline HD 131120 & 15.8 & 3.9 & 29.0 & 12.0 \\
\hline HD 105382 & 57.6 & 7.6 & 61.0 & 17.3 \\
\hline HD 46328 & 12.2 & 2.3 & 13.9 & 2.0 \\
\hline HD 16582 & 12.7 & 1.9 & 10.8 & 1.8 \\
\hline HD 111123 & 6.6 & 2.9 & 9.2 & 3.0 \\
\hline HD 74195 & 18.9 & 2.8 & 42.4 & 4.9 \\
\hline HD 181558 & 5.6 & 3.0 & 6.3 & 1.6 \\
\hline
\end{tabular}

line centre. We did not apply this tool in this work, because it is an inappropriate data manipulation when one knows that line asymmetries occur due to pulsations and/or spots, rather than being due to line blending in one part of the line for which this manipulation is justified.

Subsequently, we performed a frequency analysis of the line moments, as well as of the time series for $v \sin i(t)$ derived from the FT and for $v_{\text {macro }}(t)$ derived from the GOF. The results are shown in Figs. 6-8, where we also indicated the "true" value of $v \sin i$ as deduced from detailed line-profile modelling based on spot models or pulsation theory as given in Table 1 (horizontal lines).

For the two spotted stars, we find that the dominant frequency occurs at a value of three times the rotation frequency for both $v \sin i(t)$ and $v_{\text {macro }}(t)$. This is as expected, since these two stars each have three Si spots on their surface. As discussed by Briquet et al. (2004), the moment variations due to the spots of HD 131120 and of HD 105382 are very different from those expected for pulsations. Here, we find a correlation between the phase of $v \sin i(t)$ and the one of the second moment $\left\langle v^{2}\right\rangle(t)$, while $v_{\text {macro }}(t)$ and $\left\langle v^{2}\right\rangle(t)$ (or $\left.v \sin i(t)\right)$ show an anti-phase correlation. One needs a value of $v_{\text {macro }}(t)$ that is smaller than or close to the rotational broadening to mimic the effects of the spots on the line profile shape. That is a reflection of the fact that chemical or temperature inhomogeneities are not accompanied by velocity signatures but rather change the emergent local intensity such that the line profiles are dominantly broadened by rotation.

For all the pulsators, the three moments $\langle v\rangle,\left\langle v^{2}\right\rangle$, and $\left\langle v^{3}\right\rangle$ reveal the pulsation frequencies as already known from the previous studies of these stars. A clear result of the present study is that we recover one of the dominant mode frequencies in the time series of $v \sin i(t)$ and $v_{\text {macro }}(t)$. The pulsational velocity field due to pulsations implies periodicity in the broadening parameters $v \sin i(t)$ and $v_{\text {macro }}(t)$ when one uses only those two 
C. Aerts et al.: The Fourier transform applied to line-profile variable B stars
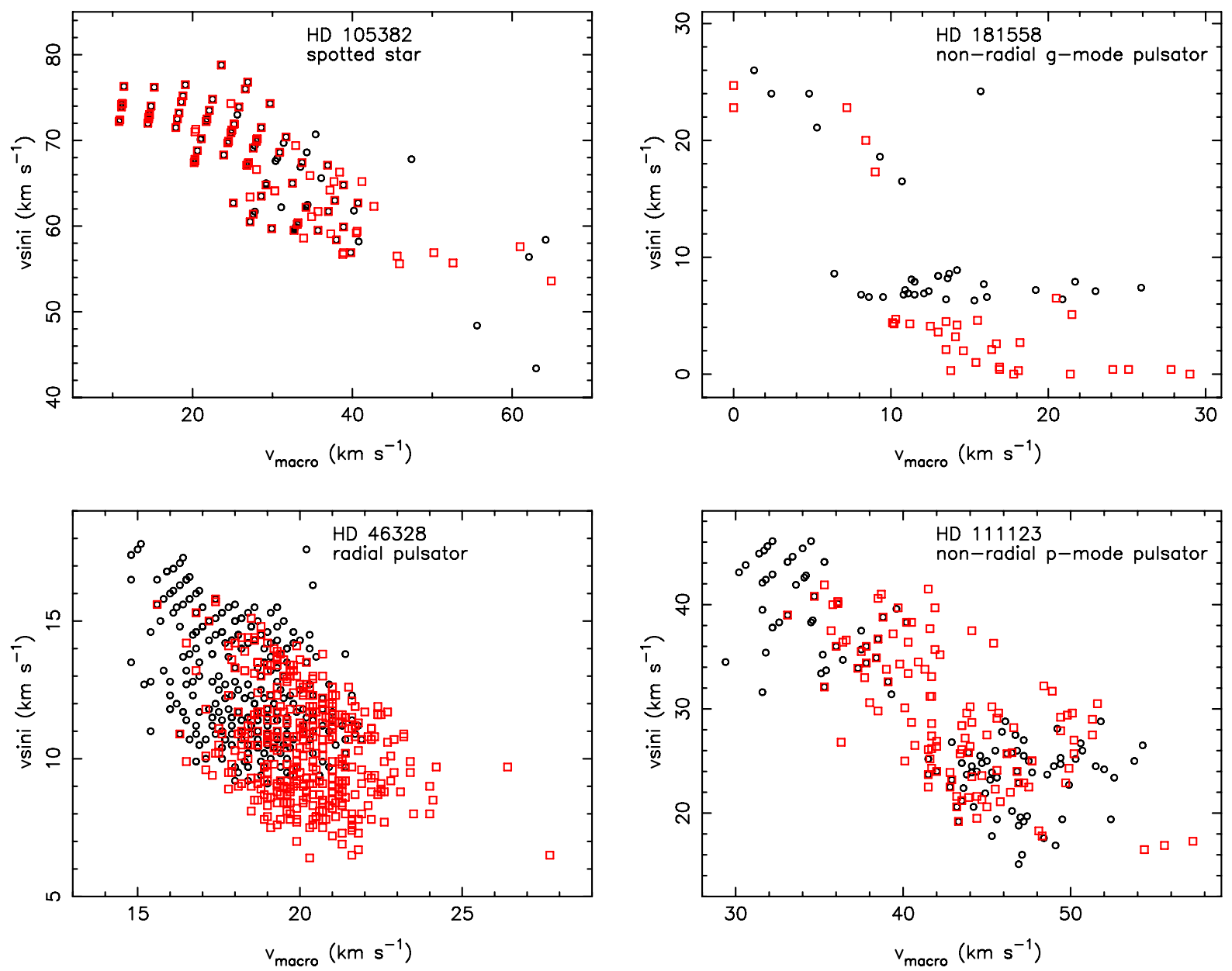

Fig. 5. Projected rotation velocity as a function of the deduced macroturbulent velocity. Black circles: $v \sin i$ deduced from the FT method and fixed for subsequent derivation of $v_{\text {macro }}$ through GOF; red squares: both values deduced from a simultaneous two-parameter GOF approach. Colour figure available in the online version of the paper.

quantities to mimic the line-profile shapes induced by the pulsations. Strong correlations between the moments and the $v \sin i(t)$ are found for the radial pulsators HD 16582 (not shown) and HD46328 (upper panel of Fig. 7), while the periodicity is less clear for these stars' $v_{\text {macro }}(t)$. For the non-radial pulsators (lower panel of Fig. 7 and both panels of Fig. 8), we again find a clear correlation between the phase behaviour of $\left\langle v^{2}\right\rangle(t)$ and $v \sin i(t)$, while $v_{\text {macro }}(t)$ has an anti-phase correlation. This reflects that the large pulsational broadening contribution in $\left\langle v^{2}\right\rangle(t)$ is compensated for as much as possible by adapting $v \sin i(t)$ to it, but this leads to line wings that are too broad given the ellipsoidal nature of the rotation profile, which is then compensated for by a smaller $v_{\text {macro }}(t)$ to represent best the shape of the pulsationally broadened line wings. At the phases where the pulsational broadening leads to lower $\left\langle v^{2}\right\rangle(t)$, i.e., smaller line width, the $v_{\text {macro }}(t)$ increases in order to mimic the broader line wings (cf. Fig. 1). As can be seen in Figs. 7 and 8, both an under- and overestimation of $v \sin i(t)$ deduced from the first zero of the FT may occur if one "downgrades" the level of the line-profile modelling by considering only the two parameters $v \sin i(t)$ and $v_{\text {macro }}(t)$ instead of the complete velocity vector due to all the detected and identified pulsation modes.

In conclusion, for both the $\mathrm{p}$ - and g-mode pulsators studied here, the anti-correlation between $v \sin i(t)$ and $v_{\text {macro }}(t)$ caused by the pulsations is clear. The macroturbulent velocities needed to mimic the pulsational broadening are close to or somewhat larger than the true rotational broadening.

\section{Conclusions}

Various high-precision time-resolved spectroscopic studies performed in the past two decades have revealed that a significant fraction of the slowly to moderately rotating $\left(v \sin i<80 \mathrm{~km} \mathrm{~s}^{-1}\right)$ B stars are line-profile variables. We have shown that periodic line-profile variability caused by surface inhomogeneities or by pulsations can sometimes be mimicked by assuming a combination of rotational and macroturbulent broadening, but only if these two parameters are allowed to take on time-dependent values. For some cases interpretation of the line profiles in terms of just $v \sin i(t)$ and $v_{\text {macro }}(t)$ is a degradation in quality for interpretating the line broadening compared to full temporal spot or pulsation modelling.

Time series analysis shows that the frequencies found in the $v \sin i(t)$ and $v_{\text {macro }}(t)$ time series coincide with the spot or pulsation frequencies that were already known to occur in the moments of the line profiles of the studied stars. Although the basic assumption of dealing with a symmetric line profile is broken, the $\mathrm{F}(\mathrm{B}) \mathrm{T}$ method remains a powerful tool for deducing $v \sin i(t)$ and subsequently fixing that value to deduce $v_{\text {macro }}(t)$ from a GOF approach, as already outlined in SDH14. A caveat is that, when the profile is particularly asymmetric, saddle points or local extrema occur in the $\mathrm{F}(\mathrm{B}) \mathrm{T}$ making the interpretation non-trivial. An appropriate estimate of $v \sin i(t)$ can then best be derived by comparing with the $\mathrm{F}(\mathrm{B}) \mathrm{T}$ results of previous or subsequent profiles in the time series, where the asymmetry is 

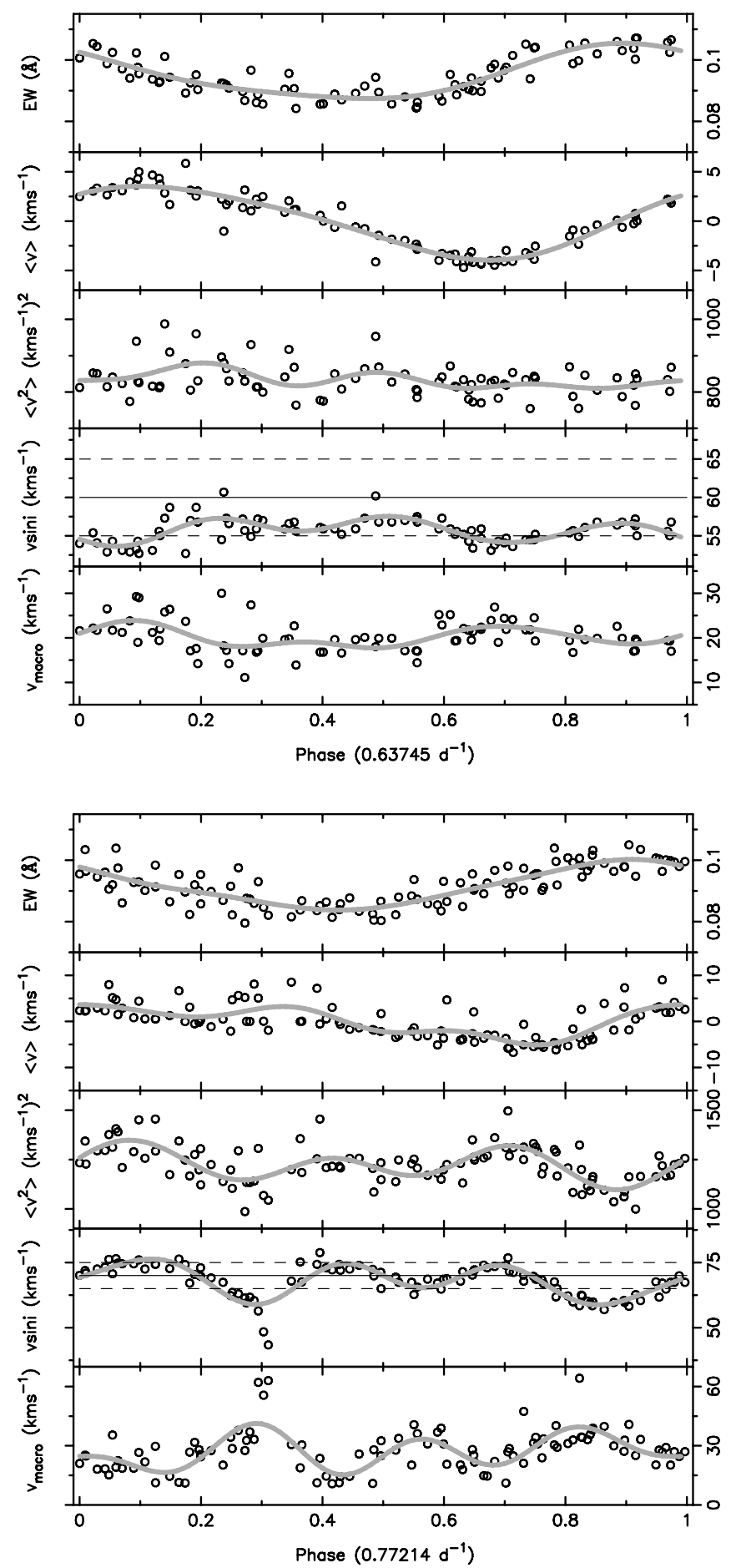

Fig. 6. Line diagnostics of two rotationally modulated variables due to $\mathrm{Si}$ spots. The horizontal lines indicate the true $v \sin i$ from spot modelling (full) and its $1 \sigma$ undertainty (dashed lines). Upper: HD 131120. Lower: HD 105382.

less during the rotation or pulsation cycle. Considering different spectral lines for the analysis may also be useful, particularly for spotted stars with chemical inhomogeneities. Pulsations tend to affect all metal lines similarly unless the magnetic pressure dominates the gas pressure in the line-forming regions.

Our study shows that one should not rely on a single snapshot spectrum to derive accurate average values of $v \sin i$ and $v_{\text {macro }}$ for main-sequence stars with slow or moderate rotation, because the effect of spots, and of pulsations in particular, implies that a relatively large range of these two parameters is necessary to
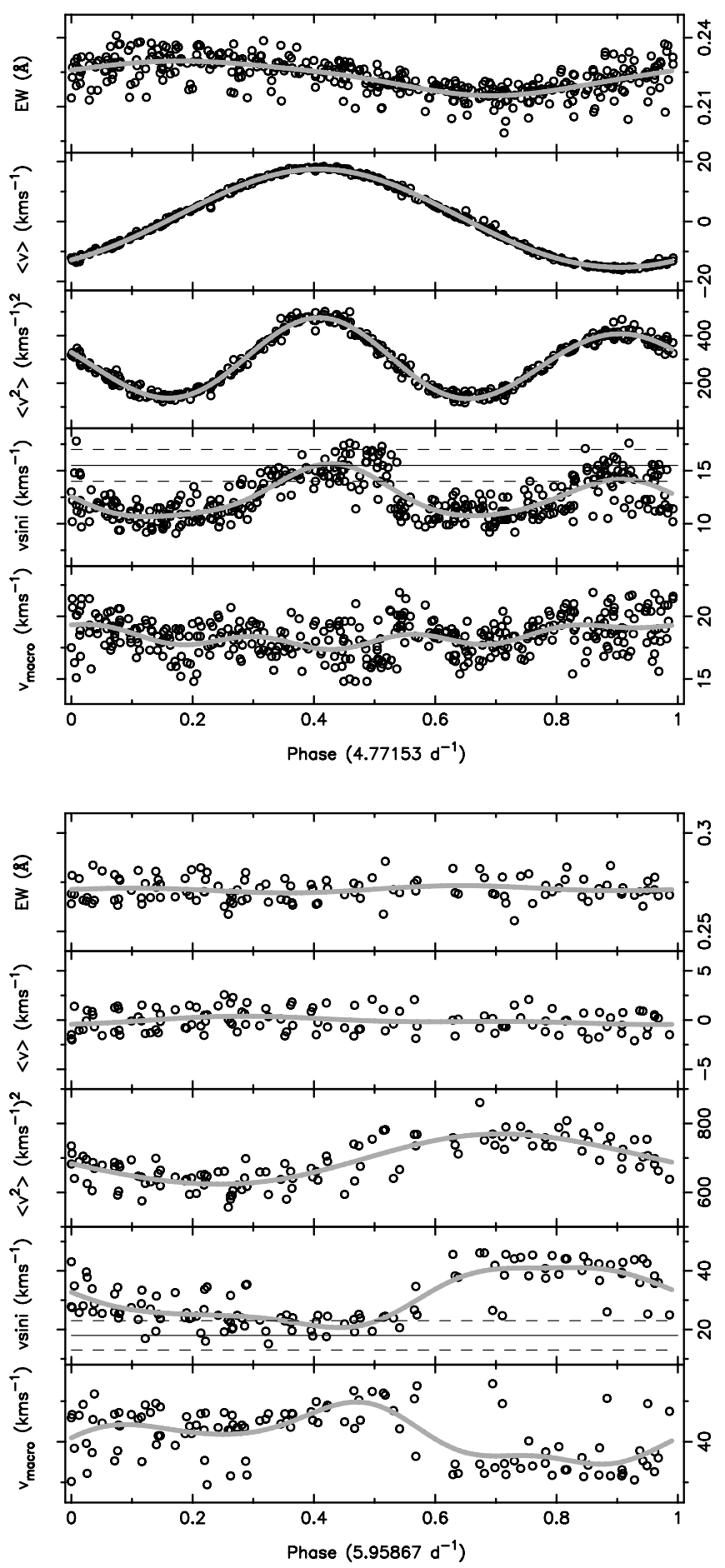

Fig. 7. Line diagnostics of two p-mode pulsators. Upper: HD 46328, which is a monoperiodic radial pulsators. Lower: HD 111123, which is a multiperiodic non-radial pulsator. The horizontal lines indicate the true $v \sin i$ from modelling of the line-profile variations in terms of pulsation theory (full) and its $1 \sigma$ undertainty (dashed lines).

describe the profile changes over the variability cycles. We have shown that ignoring the time-variable nature of the line profiles

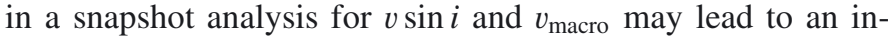
correct interpretation of the derived values of both quantities. In particular, a high value of $v_{\text {macro }}$ may just occur because the pulsational character of the star is not taken into account. The cause of macroturbulent velocities occurring in line-profile variable O-type stars and B-type supergiants remains unclear and 
C. Aerts et al.: The Fourier transform applied to line-profile variable B stars
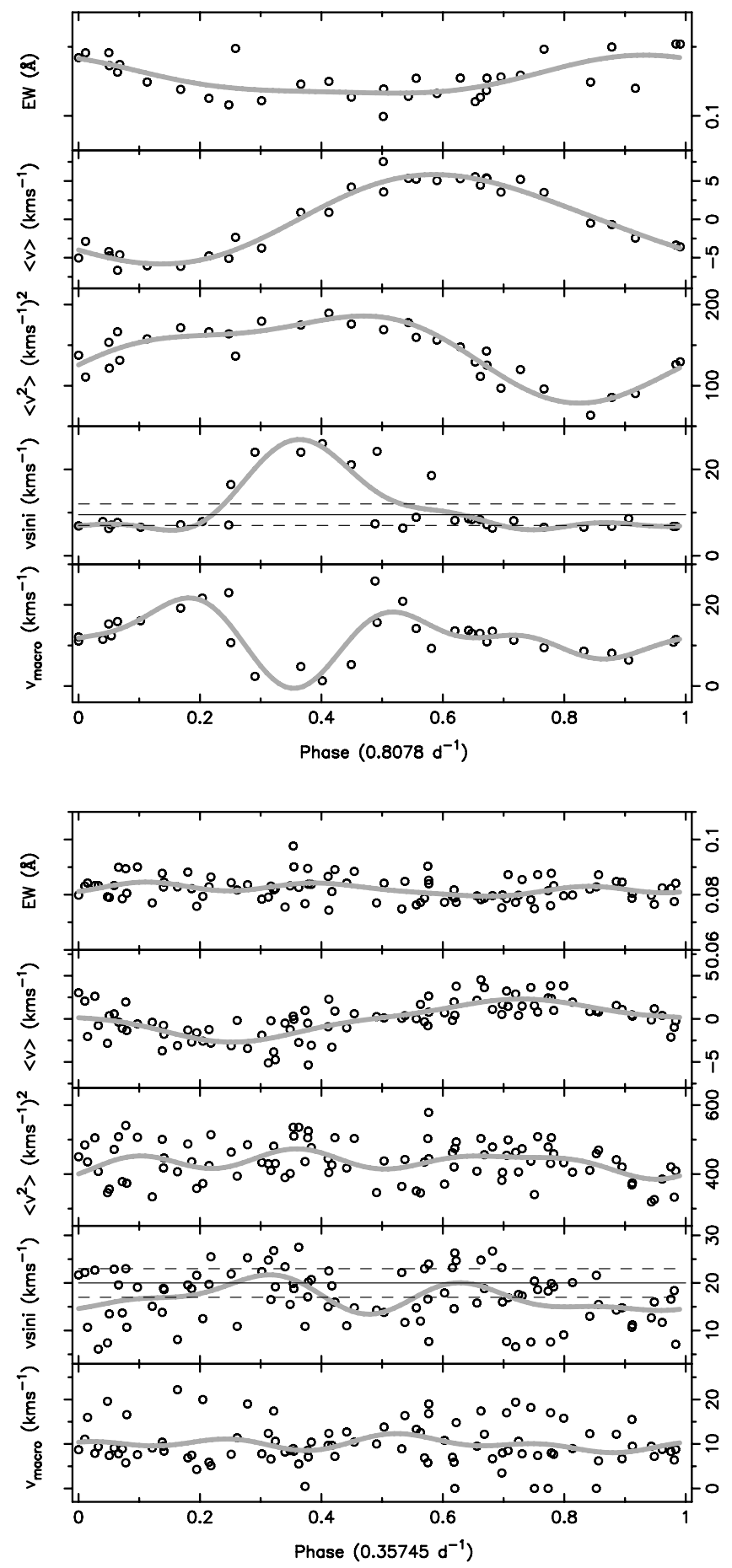

Fig. 8. Line diagnostics of two g-mode pulsators. Upper: HD 181558 , which has a dominant $g$ mode. Lower: HD 74195, which has various $g$ modes of almost equal amplitude. The horizontal lines indicate the true $v \sin i$ from modelling of the line-profile variations in terms of pulsation theory (full) and its $1 \sigma$ undertainty (dashed lines).

may be quite different from the case of dwarf stars considered here, as the combined studies by Simón-Díaz et al. (2010) and SDH2014 have shown.

In a follow-up paper, we plan to present a simulation study where all the effects that cause a deviation from symmetry of spectral line profiles will be evaluated in terms of their influence on the derivation of $v \sin i$ from $\mathrm{F}(\mathrm{B}) \mathrm{T}$ and on $v_{\text {macro }}$. The amount of macroturbulent broadening needed to mimic spots or pulsations depends not only on the brightness or chemical contrasts in

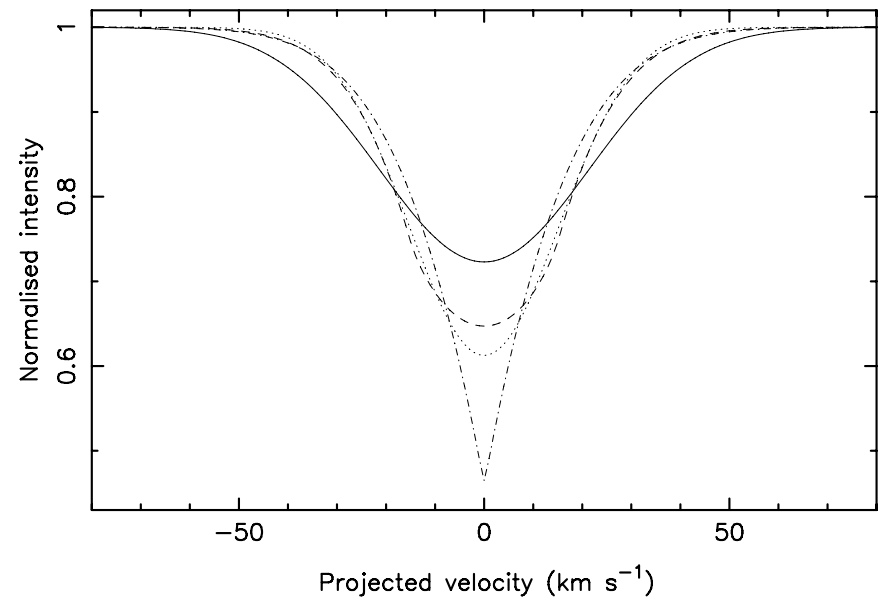

Fig. 9. Simulated line profiles of equal equivalent width for $v \sin i=$ $15 \mathrm{~km} \mathrm{~s}^{-1}$ and a macroturbulent velocity of $20 \mathrm{~km} \mathrm{~s}^{-1}$ for an isotropic (full line), a pure radial (dashed line), a pure tangential (dashed-dot line), and a radial-tangential with equal contributions of them both (dotted line).

the case of spots or on the amplitudes and spherical wavenumbers $(\ell, m)$ of the modes in case of pulsations, but also on the inclination angle of the star. Moreover, the asymmetry may not only come from spots or pulsations as discussed here, but also from, for example, gravity darkening, deformation from a spherical shape because of fast rotation and/or binarity, reflection effects in close binaries, spectral line blending, etc. In addition, the level of the radial and tangential contributions to the macroturbulent velocity must be varied from the standard case where they are taken as equal if the aim is to get a complete picture and solid predictive recipes for $v_{\text {macro }}$. This is illustrated in Fig. 9 where we compare simulated profiles for $v \sin i=15 \mathrm{~km} \mathrm{~s}^{-1}$ and an isotropic, a pure radial, a pure tangential, and an equal-share radial-tangential macroturbulent velocity of $20 \mathrm{~km} \mathrm{~s}^{-1}$. It can be seen that the nature of the macroturbulent velocity has a strong effect on the profile shape. That p-mode pulsations typically correspond to a factor 10 to 100 larger radial than tangential velocity, while it is the other way around for g-mode pulsations (Aerts et al. 2010), implies that restricting $v_{\text {macro }}$ to an equal share of the radial and tangential contributions, as mostly done in the literature so far, is not optimal. This issue has already been addressed in Aerts et al. (2009) where profiles were modelled for the three extremes (isotropic, radial, tangential) of macroturbulence as also shown in Fig. 9. Only an extensive simulation study in which all the phenomena that affect the line profile shape are addressed can lead to a full assessment of the appropriateness, or not, of using only $v \sin i$ and $v_{\text {macro }}$ estimates rather than complete line-profile modelling based on solid theoretical descriptions, in evaluating theoretical stellar evolution models.

Acknowledgements. This work was initiated by discussions among the four authors during the 2013 International Francqui Symposium "What Asteroseismology has to offer to Astrophysics" (fys.kuleuven.be/ ster/meetings/francqui/francqui) funded by and organised under the auspices of the Francqui Foundation of Belgium. This symposium also marked the end of the Advanced Grant: "Probing Stellar Physics and Testing Stellar Evolution through Asteroseismology" (PROSPERITY, www.ster.kuleuven.be/PROSPERITY) awarded to the first author by the European Research Council under the European Community's Seventh Framework Programme (FP7/2007-2013)/ERC grant agreement n²27224. S.S.-D. acknowledges funding by [a] the Spanish Ministry of Economy and Competitiveness (MINECO) under the grants AYA2010-21697-C05-04 and Severo Ochoa SEV-2011-0187, and [b] the Canary Islands Government under grant PID2010119. This research has made use of the SIMBAD database, operated at the CDS, Strasbourg, France. 


\section{References}

Aerts, C., \& De Cat, P. 2003, Space Sci. Rev., 105, 453

Aerts, C., de Pauw, M., \& Waelkens, C. 1992, A\&A, 266, 294

Aerts, C., De Cat, P., Cuypers, J., et al. 1998, A\&A, 329, 137

Aerts, C., Marchenko, S. V., Matthews, J. M., et al. 2006, ApJ, 642, 470

Aerts, C., Puls, J., Godart, M., \& Dupret, M.-A. 2009, A\&A, 508, 409

Aerts, C., Christensen-Dalsgaard, J., \& Kurtz, D. W. 2010, Asteroseismology (Heidelberg: Astron. Astrophys. Lib., Springer)

Aerts, C., Molenberghs, G., Kenward, M. G., \& Neiner, C. 2014, ApJ, 781, 88

Alecian, E., Kochukhov, O., Neiner, C., et al. 2011, A\&A, 536, L6

Ammler-von Eiff, M., \& Reiners, A. 2012, A\&A, 542, A116

Asplund, M., Ludwig, H.-G., Nordlund, Å., \& Stein, R. F. 2000, A\&A, 359, 669

Bagnulo, S., Landstreet, J. D., Fossati, L., \& Kochukhov, O. 2012, A\&A, 538,

A129

Briquet, M., \& Aerts, C. 2003, A\&A, 398, 687

Briquet, M., Aerts, C., Lüftinger, T., et al. 2004, A\&A, 413, 273

Briquet, M., Hubrig, S., De Cat, P., et al. 2007a, A\&A, 466, 269

Briquet, M., Hubrig, S., Schöller, M., \& De Cat, P. 2007b, Astron. Nachr., 328, 41

Briquet, M., Neiner, C., Aerts, C., et al. 2012, MNRAS, 427, 483

Brott, I., Evans, C. J., Hunter, I., et al. 2011, A\&A, 530, A116

Campos, A. J., \& Smith, M. A. 1980, ApJ, 238, 250

Carroll, J. A. 1933, MNRAS, 93, 478

Claret, A., \& Bloemen, S. 2011, A\&A, 529, A75

Cuypers, J., Aerts, C., Buzasi, D., et al. 2002, A\&A, 392, 599

De Cat, P., \& Aerts, C. 2002, A\&A, 393, 965

De Cat, P., Briquet, M., Daszyńska-Daszkiewicz, J., et al. 2005, A\&A, 432, 1013

De Ridder, J., Dupret, M.-A., Neuforge, C., \& Aerts, C. 2002, A\&A, 385, 572

Degroote, P., Aerts, C., Baglin, A., et al. 2010, Nature, 464, 259

Degroote, P., Acke, B., Samadi, R., et al. 2011, A\&A, 536, A82

Degroote, P., Aerts, C., Michel, E., et al. 2012, A\&A, 542, A88

Dravins, D., Lindegren, L., \& Torkelsson, U. 1990, A\&A, 237, 137
Gray, D. F. 2005, The Observation and Analysis of Stellar Photospheres, 3rd edn. (Cambridge University Press)

Groot, P. J., Piters, A. J. M., \& van Paradijs, J. 1996, A\&AS, 118, 545

Howarth, I. D. 2004, in Stellar Rotation, eds. A. Maeder, \& P. Eenens, IAU Symp., 215, 33

Hubrig, S., Briquet, M., Schöller, M., et al. 2006, MNRAS, 369, L61

Hubrig, S., Briquet, M., De Cat, P., et al. 2009, Astron. Nachr., 330, 317

Hubrig, S., Ilyin, I., Briquet, M., Schöller, M., \& Mathys, G. 2013, in ASP Conf. Ser. 479, eds. H. Shibahashi, \& A. E. Lynas-Gray, 121

Hunter, I., Brott, I., Langer, N., et al. 2009, A\&A, 496, 841

Jankov, S. 1995, Publications de l'Observatoire Astronomique de Beograd, 50, 75

Maeder, A. 2009, Physics, Formation and Evolution of Rotating Stars (Heidelberg: Astron. Astrophys. Lib., Springer)

Markova, N., Puls, J., Simón-Díaz, S., et al. 2014, A\&A, 562, A37

Mathias, P., Aerts, C., de Pauw, M., Gillet, D., \& Waelkens, C. 1994, A\&A, 283, 813

Piters, A. J. M., Groot, P. J., \& van Paradijs, J. 1996, A\&AS, 118, 529

Reiners, A., \& Schmitt, J. H. M. M. 2002, A\&A, 388, 1120

Reiners, A., \& Schmitt, J. H. M. M. 2003, A\&A, 412, 813

Saesen, S., Briquet, M., \& Aerts, C. 2006, Commun. Asteroseismol., 147, 109

Schrijvers, C., \& Telting, J. H. 1999, A\&A, 342, 453

Simón-Díaz, S., \& Herrero, A. 2014, A\&A, 562, A135 (SDH14)

Simón-Díaz, S., Herrero, A., Uytterhoeven, K., et al. 2010, ApJ, 720, L174

Slettebak, A. 1949, ApJ, 110, 498

Smith, M. A., \& Gray, D. F. 1976, PASP, 88, 809

Smith, M. A., \& Karp, A. H. 1978, ApJ, 219, 522

Sundqvist, J. O., Simón-Díaz, S., Puls, J., \& Markova, N. 2013, A\&A, 559, L10

Telting, J. H., Aerts, C., \& Mathias, P. 1997, A\&A, 322, 493

Telting, J. H., Schrijvers, C., Ilyin, I. V., et al. 2006, A\&A, 452, 945

Uytterhoeven, K., Briquet, M., Aerts, C., et al. 2005, A\&A, 432, 955

Zima, W. 2008, Commun. Asteroseismol., 155, 17 